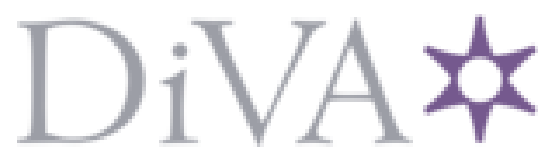

http://www.diva-portal.org

This is the published version of a paper published in Alzheimer's \& Dementia.

Citation for the original published paper (version of record):

Sindi, S., Håkansson, K., Hagman, G., Kulmala, J., Soininen, H. et al. (2014)

Mid-life work-related stress increases dementia risk in late-life: The CAIDE 30-year study.

Alzheimer's \& Dementia, 10(4, Supplement): P746

http://dx.doi.org/10.1016/j.jalz.2014.05.1408

Access to the published version may require subscription.

N.B. When citing this work, cite the original published paper.

Permanent link to this version:

http://urn.kb.se/resolve?urn=urn:nbn:se:Inu:diva-51881 


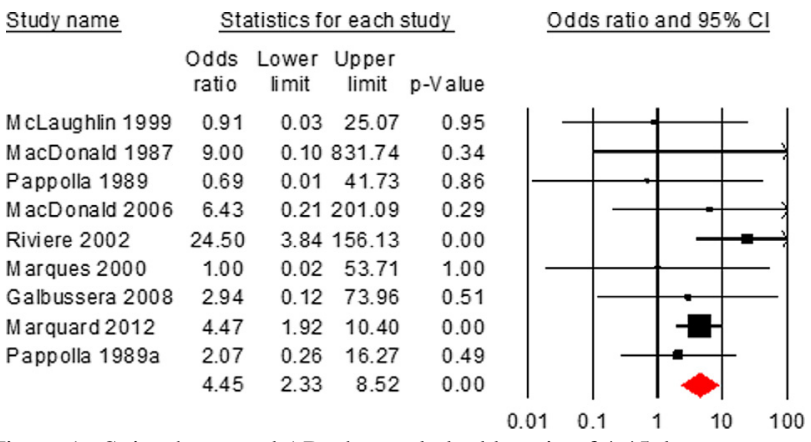

Figure 1. Spirochetes and AD: the pooled odds ratio of 4.45 demonstrates a statistically significant association of spirochetes with $\mathrm{AD}(\mathrm{p}<0.05)$.

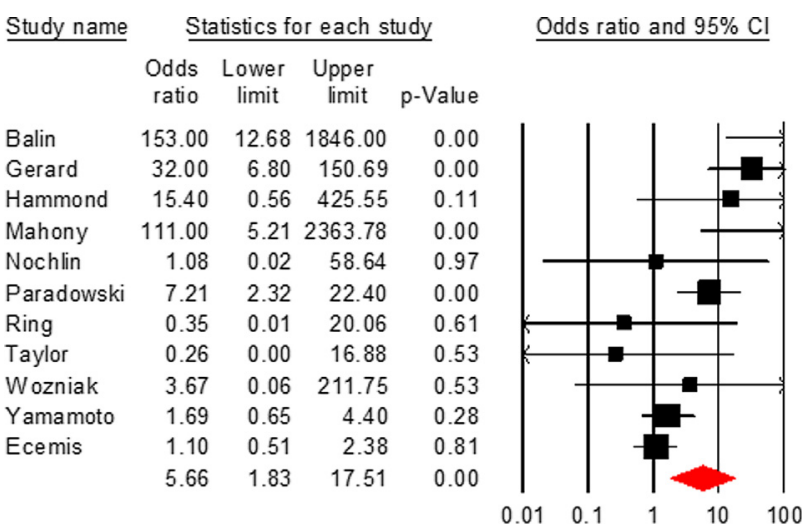

Figure 2. Cpn and AD: the pooled odds ratio of 5.66 demonstrates a statistically significant association of $\mathrm{Cpn}$ with $\mathrm{AD}(\mathrm{p}<0.05)$.

\section{P3-315 MID-LIFE WORK-RELATED STRESS INCREASES DEMENTIA RISK IN LATE-LIFE: THE CAIDE 30-YEAR STUDY}

Shireen Sindi ${ }^{1}$, Krister Hakansson ${ }^{2}$, Göran Hagman ${ }^{1}$, Jenni Kulmala ${ }^{3}$, Hilkka Soininen ${ }^{4}$, Ingemar Kareholt ${ }^{5}$, Alina Solomon ${ }^{4}$, Miia Kivipelto ${ }^{6}$, ${ }^{1}$ Karolinska Institutet, Växjö, Sweden; ${ }^{2}$ Karolinska Institutet, Stockholm, Sweden; ${ }^{3}$ University of Jyväskylä, Jyväskylä, Finland; ${ }^{4}$ University of Eastern Finland, Kuopio, Finland; ${ }^{5}$ Karolinska Institute, Stockholm, Sweden; ${ }^{6}$ Karolinska Institutet, Stockholm, Finland.

Contacte-mail: shireen.sindi@ki.se

Background: The associations between work-related stress and various health outcomes in mid-life are well documented, yet less is known about the effects on late-life cognitive process and dementia. The current study investigated the associations between work-related stress in mid-life and the development of cognitive impairment and Alzheimer's disease in late-life. Methods: The data was derived from the Cardiovascular Risk Factors, Aging, and Incidence of Dementia (CAIDE) study; a prospective cohort study. Participants were randomly selected from four independent population-based samples that completed cardiovascular surveys. First baseline examinations occurred when participants were 50 years old on average, in 1972, 1977, 1982, or 1987. A random sample of 2,000 individuals was selected for re-examinations (carried out in 1998 and 2005-2008), where 1,511 subjects participated in at least one re-examination. The reexaminations included an extensive neuropsychological and cognitive assessment. Follow-up time was on average $28($ S.E.M. $=0.17$ ) years. Work-related stress comprised the total score of two questions administered in mid-life. The questions asked participants to rate their stress related to meeting demands at work, and constant hurry at work. Groups were categorized so that those with high or medium levels of stress were compared to those with low levels or no work-related stress. Results: High levels of work-related stress in mid-life were associated with higher risk of cognitive impairment (where participants with cognitive impairment and dementia were compared to the group with no cognitive impairment) [odds ratio (OR), 1.5; 95\% confidence interval (CI), 1.1-2.1], and Alzheimer's disease [OR, 2.1; CI, 1.1-3.9], when assessed at the first or second follow-up. Results remained significant after adjusting for age, education, marital status, chronic health conditions, apolipoprotein E $\varepsilon 4$ allele (APOE $\varepsilon 4$ ), measures of hopelessness and loneliness. Conclusions: High levels of mid-life work-related stress predict the risk of developing dementia in late-life. The evidence suggests that individuals experiencing high levels of work-related stress form an important at-risk population. Preventive interventions are needed for this population in order to postpone or prevent the development of cognitive impairment and Alzheimer's disease.

\section{P3-316 CALIBRATING LONGITUDINAL COGNITIVE PERFORMANCE ACROSS DIVERSE NEUROPSYCHOLOGICAL BATTERIES AND DATASETS}

Alden L. Gross ${ }^{1}$, Shubhabrata Mukherjee ${ }^{2}$, Richard Sherva ${ }^{3}$, John Kauwe ${ }^{4}$, Stephen Jeffrey Newhouse ${ }^{5}$, Leo Waterston ${ }^{6}$, Richard Norman Jones ${ }^{7}$, Robert Green ${ }^{8}$, Paul Crane ${ }^{9},{ }^{1}$ Johns Hopkins Bloomberg School of Public Health, Baltimore, Maryland, United States; ${ }^{2}$ University of Washington School of Medicine, Seattle, Washington, United States; ${ }^{3}$ Boston University, Boston, Massachusetts, United States; ${ }^{4}$ Brigham Young University, Provo, Utah, United States; ${ }^{5}$ KCL, London, United Kingdom; ${ }^{6}$ Brigham and Women's Hospital and Harvard Medical School, Boston, Massachusetts, United States, ${ }^{7}$ Institute for Aging Research, Hebrew SeniorLife, Boston, Massachusetts, United States; ${ }^{8}$ Division of Genetics, Department of Medicine, Brigham and Women's Hospital and Harvard Medical School, Boston, MA; ${ }^{9}$ University of Washington, Seattle, Washington, United States. Contact e-mail: smukherj@uw.edu

Background: To identify the optimal approach for calibrating longitudinal cognitive performance across studies with different neuropsychological batteries to use as a common phenotype in genetic research on Alzheimer's disease (AD). Methods: We examined four approaches to calibrate cognitive performance in eight large longitudinal studies of AD (N=10,997): (1) selecting a common test across datasets, (2) standardizing and averaging together all tests, (3) confirmatory factor analysis (CFA) with continuous indicators, and (4) CFA with categorical indicators. Datasets included the Alzheimer's Disease Neuroimaging Initiative (ADNI), Rush Memory and Aging Project, Religious Orders Study, Cache County Study on Memory and Aging, Myriad Tarenflurbil phase III clinical trial, AddNeuroMed, Adult Changes in Thought, and the National Alzheimer's Disease Coordinating Center. To compare precision, we determined minimum sample sizes necessary to detect a $25 \%$ decline with $80 \%$ power using each approach. To compare criterion validity, we examined the association of cognitive change using each approach with changes over up to six years in whole brain cortical thickness and hippocampal volume using available MRI data from ADNI in joint process latent growth curve models. Results: Summary cognitive measures were highly correlated (all r's $>0.88$ ). CFA with categorical indicators required the lowest sample size to detect $25 \%$ cognitive decline with $80 \%$ power $(\mathrm{N}=253)$ compared to the common test $(\mathrm{N}=283)$, standardize and average ( $\mathrm{N}=300)$, and CFA with continuous indicators $(\mathrm{N}=329)$ approaches. Associations with changes in whole brain cortical thickness and hippocampal volume were strongest for the CFA with categorical indicators approach $(Z=7.0 ; Z=5.2$, respectively) compared to the common test $(Z=6.2 ; Z=4.5)$, standardize and average $(Z=5.5 ; Z=3.7)$, and CFA with continuous indicators $(Z=6.1 ; Z=4.1)$ approaches. Conclusions: CFA with categorical indicators demonstrated the best precision and thus greater power to detect change. It also was more strongly correlated with biological markers than other approaches. This approach has wide applicability to directly compare cognitive performance across studies, making it a good operational phenotype for genetic analyses of cognitive decline in $\mathrm{AD}$. 